\title{
Operators commuting with multi-parameter shift semigroups
}

\section{OleH LOPUSHANSKY and SERGII SHARYN}

\section{ABSTRACT.}

Using operators of cross-correlation with ultradistributions supported by a positive cone, we describe a commutative algebra of shift-invariant continuous linear operators, commuting with contraction multi-parameter semigroups over a Banach space. Thereby, we generalize classic Schwartz's and Hörmander's theorems on shift-invariant operators.

Acknowledgements. The authors would like to thank the referee for valuable comments which helped to improve the manuscript.

\section{REFERENCES}

[1] Butzer, P. L. and Berens, H., Semi-Group of Operators and Approximation, Springer-Verlag, New-York, 1967

[2] Colzani, L. and Sjögren, P., Translation-invariant operators on Lorentz spaces $L(1, q)$ with $0<q<1$, Studia Math., 132 (1999), No. 2, 101-124

[3] Dales, H. G. and Millinoton, A., Translation-invariant linear operators, Math. Proc. Cambridge Phil. Soc., 113 (1993), No. 1, 161-172

[4] Feichtinger, H. G., Fëuhr, H., Grëochenig, K. and Kaiblinger, N., Operators commuting with a discrete subgroup of translations, J. Geometric Analysis, 16 (2006), No. 1, 53-67

[5] Grafakos, L. and Soria, J., Translation-invariant bilinear operators with positive kernels, Integr. Equ. Oper. Theory, 66 (2010), 253-264

[6] Grothendieck, A., Produits tensoriels topologiques et espaces nucléaries, Mem. Amer. Math. Soc., 16, 1955

[7] Hille, E. and Phillips, R., Functional analysis and semi-hroups, AMS Coll. Publ., vol.XXXI, New York, 1957

[8] Hörmander, L, Estimates for transtation invariant operators in $L^{p}$ spaces, Acta Math., 104 (1960), No. 1, 93-140

[9] Hytönen, T., Translation-invariant operators on spaces of vector-valued functions, Helsinki University of Technology, 2003

[10] Jarchow, H., Locally Convex Spaces, Teubner, Stuttgart, 1981

[11] Komatsu, H., An Introduction to the theory of generalized functions, Tokyo University Publ., 2000

[12] Lopushansky, O. and Sharyn, S., Polynomial ultradistributions on cone $\mathbb{R}_{+}^{d}$, Topology, 48 (2009), No. 2-4, 80-90

[13] Raikov, D. A., Double closed-graph theorem for topological linear spaces, Siber. Math. J., 7 (1967), No. 2, 287-300

[14] Schaefer, H., Topological vector spaces, Springer-Verlag, New-York, 1971

[15] Schwartz, L., Theorie des distributions, Hermann, Paris, 1966

[16] Seeley, R. T., Extensions of $C^{\infty}$-functions defined in a half-space, Proc. Amer. Math. Soc., 15 (1964), 625-626

[17] Tovstolis, A., On translation invariant operators in Hardy spaces in tube domains over open cones, Methods Funct. Anal. Topology, 9 (2003), No. 3, 262-272

[18] Swartz, C. W., Translation invariant linear operators and generalized functions, Czechoslovak Math. J., 25 (1975), No. 2, 202-213

[19] Weiss, G., Representation of shift-invariant operators on $L^{2}$ by $H^{\infty}$ transfer functions: An elementary proof, a generalization to $L^{p}$, and a counterexample for $L^{\infty}$, Math. Control Signals Systems, 4 (1991), 193-203

Received: 22.04.2013; In revised form: 16.10.2013; Accepted: 15.01.2014

2010 Mathematics Subject Classification. 46F05, 46H30, 47A60.

Key words and phrases. Shift-invariant operator, ultradistribution, multi-parameter semigroup.

Corresponding author: Sharyn Sergii; sharyn.sergii@gmail.com 
UNIVERSITY OF RZESZÓW

FACULTY OF MATHEMATICS AND NATURAL SCIENCES

16A ReJTANA, 35-959 RzESzÓW, POLAND

E-mail address: ovlopusz@ur. edu.pl

Department of Mathematics AND COMPUter Sciences

PRECARPATHIAN NATIONAL UNIVERSITY

57 SHEVCHENKA, 76-018 IVANO-FRANKIVSK, UKRAINE

E-mail address: sharyn.sergii@gmail.com 